\title{
Normal Saccadic Responses Using Video Head Impulse Test In Healthy Young Adults
}

Nurul Amira Omar ${ }^{1}$, Saiful Adli bin Jamaluddin ${ }^{1}$

${ }^{1}$ Department of Audiology and Speech-Language Pathology, Kulliyyah of Allied Health Sciences, International Islamic University Malaysia

\section{Presenter: Nurul Amira Omar}

Introduction: Vertigo and dizziness are common symptoms reported in audiology and ENT clinics. One of the objective assessments includes the video head impulse test (vHIT) where gain \& amp; velocity responses of eye movements relative to the head movements are recorded using an infrared camera. Materials and Methods: Thirty normal hearing subjects age between 18 to 25 years old participated in this study. Exclusion factors include those with the history of head or neck injury and vertigo. At least ten lateral, left anterior right posterior (LARP), and right anterior left posterior (RALP) responses were recorded for each participant by making small unpredictable head movements. Results: The average velocity gain for lateral responses at $40 \mathrm{~ms}, 60 \mathrm{~ms}$ and $80 \mathrm{~ms}$ were $1.05 \pm 0.003,1.03 \pm 0.002$ and $1.01 \pm$ 0.003 respectively. The LARP and RALP average velocity regression were $1.01 \pm$ 0.24 for left anterior and $1.05 \pm 0.25$ for right posterior, $1.08 \pm 0.31$ for right anterior and $1.12 \pm 0.30$ for left posterior. One sample T-test was conducted to compare lateral responses to a previous study by Mossman et al. 2015. There were significant differences in velocity gain at $60 \mathrm{~ms}$ and $80 \mathrm{~ms}$ where, t $(59)=5.56, \mathrm{p}<$ 0.01 and $t(59)=2.86, p<0.01$, respectively. Conclusion: This indicates the importance of establishing norms for clinics as various factors could affect the results such as techniques used and equipment differences. A follow-up study on subjects with vestibular disorders is required to validate this data as a normative reference. 